\title{
Tratamiento de aguas residuales con colorantes reactivos mediante proceso de oxidación avanzada Fenton
} y variantes

\section{Wastewater treatment with reactive dyes by advanced oxidation process Fenton and variants.}

\author{
Fernando Anaya Meléndez ${ }^{3 *}$
}

\section{RESUMEN}

Se ha realizado el estudio comparativo del tratamiento del colorante reactivo Sunfix Yellow S4GL (RAS) mediante proceso de oxidación avanzada Fenton y $\mathrm{H}_{2} \mathrm{O}_{2} / \mathrm{UV}$. Este colorante se encuentra presente en aguas residuales de Tintorería en la Industria Textil. Los valores óptimos aplicando el método $\mathrm{H}_{2} \mathrm{O}_{2} / \mathrm{UV}$ fueron, $\left[\mathrm{H}_{2} \mathrm{O}_{2}\right]=1292 \mathrm{mg} / \mathrm{L}$; $\mathrm{pH}=3$ y 3 lámparas UVC con las cuales se consiguió una decoloración del $100 \%$ en 40 min de exposición a la luz UVC. En el caso del método Fotofenton, los valores óptimos hallados fueron, $\left[\mathrm{Fe}^{+2}\right]=3,52 \mathrm{mg} / \mathrm{L},\left[\mathrm{H}_{2} \mathrm{O}_{2}\right]=300 \mathrm{mg} / \mathrm{L}$, $\mathrm{pH}=2,51$ y 2 lámparas UVC, con las cuales se consiguió una decoloración del $100 \%$ en 30 min de exposición a la luz UV-C.El método Fotofenton es más eficiente que el $\mathrm{H}_{2} \mathrm{O}_{2} / \mathrm{UV}$ pues emplea menor cantidad de $\mathrm{H}_{2} \mathrm{O}_{2}$, menor potencia de radiación y logra la decoloración del $100 \%$ en menor tiempo.

Palabras claves: Proceso de oxidación avanzada, colorante amarillo Sunfix S4GL (CAS), Fotofenton, $\mathrm{H}_{2} \mathrm{O}_{2} / \mathrm{UV}$, decoloración.

\begin{abstract}
The comparative study of the Sunfix Yellow S4GL (RAS) reactive dye treatment was carried out using the advanced oxidation process Fenton and $\mathrm{H} 2 \mathrm{O} 2$ / UV. This dye is present in sewage wastewater in the Textile IndustryThe optimal values applying the $\mathrm{H} 2 \mathrm{O} 2$ / UV method were, [H2O2] $=1292 \mathrm{mg} / \mathrm{L} ; \mathrm{pH}=3$ and $3 \mathrm{UVC}$ lamps with which a $100 \%$ discoloration was achieved in $40 \mathrm{~min}$ of exposure to UVC light.In the case of the Fotofenton method, the optimal values found are $[\mathrm{Fe}+2]=3.52 \mathrm{mg} / \mathrm{L},[\mathrm{H} 2 \mathrm{O} 2]=300 \mathrm{mg} / \mathrm{L}, \mathrm{pH}=2.51$ and 2 UVC lamps with which a $100 \%$ discoloration was achieved in 30 min exposure to UV-C light. The Fotofenton method is more efficient than $\mathrm{H} 2 \mathrm{O} 2$ / UV because it uses less $\mathrm{H} 2 \mathrm{O} 2$, less radiation power and achieves $100 \%$ discoloration in less time.
\end{abstract}

Keywords: Advanced oxidation process, Sunfix S4GL yellow color(CAS), Fotofenton, H2O2 / UV, discoloration. 


\section{INTRODUCCIÓN}

Los colorantes empleados en el proceso de tintorería son diversos, entre naturales y artificiales. Dentro de los colorantes artificiales se encuentra el colorante tipo reactivo que es el más empleado, y presentan niveles de reactividad (alta, media y baja) los que depende del grupo reactivo presente en su molécula. Los grupos reactivos presentes en la molécula del colorante son en muchos casos compuestos persistentes que al ser vertidos a los cuerpos receptores en la última etapa del proceso representan un peligro para el medio ambiente. Los contaminantes persistentes por presentar características fisicoquímicas especiales no pueden ser eliminados mediante los tratamientos tradicionales (físicos, químicos y biológicos). El caso de los colorantes tipo azo, dentro de la industria textil, merece una consideración especial si se tiene en cuenta que al menos el 70\% de las 106 toneladas producidas anualmente pertenecen a esta categoría (Hai et al., 2007). Adicionalmente, los colorantes azo presentan rangos de fijación variable (del orden de 80$95 \%$ ), por lo que se esperan pérdidas de colorante en el efluente textil en un intervalo del 5 al 20\% en el caso de colorantes azo tipo ácido, y hasta un 50\% para los de tipo reactivo (Van der Zee et al., 2005).

La descarga de tales efluentes resulta problemática, no sólo atendiendo a las razones estéticas, sino a los efectos negativos causados por los productos de degradación que pueden resultar tóxicos y mutagénicos para la vida acuática y los seres humanos (Ahn, et al., 1999). Los procesos de oxidación avanzada (POAs) se perfilan como una de las alternativas más atractivas desde el punto de vista técnico y ambiental para la degradación de efluentes tóxicos debido a su versatilidad, ya que existen diferentes alternativas para la generación de los radicales $\mathrm{HO}$ que pueden ser adaptadas a situaciones específicas (Perez et al., 2002; Malato-Rodríguez et al., 2004).

La presente investigación cumplió el objetivo general de lograr las condiciones óptimas de degradación del colorante amarillo Sunfix S4GL (CAS) mediante la aplicación de los métodos Fotofenton y $\mathrm{H}_{2} \mathrm{O}_{2} / \mathrm{UV}$.

\section{MATERIALES Y MÉTODOS}

El estudio sigue la metodología de investigación aplicada de tipo experimental para el tratamiento del colorante amarillo Sunfix S4GL (CAS).

\section{Reactivos}

- Colorante amarillo Sunfix S4GL (CAS), estado sólido.

- Solución de peróxido de hidrógeno $\mathrm{H}_{2} \mathrm{O}_{2}$ al $50 \%, \mathrm{~V} / \mathrm{V}$

- Solución de $\mathrm{FeSO}_{4} .7 \mathrm{H}_{2} \mathrm{O} ; 6.298 \mathrm{mM}$.

- Soluciones diluidas de $\mathrm{H}_{2} \mathrm{SO}_{4}$ y $\mathrm{NaOH}$ para regular el pH de la muestra.

\section{Equipos}

- Agitador magnético

- Potenciómetro TOA-HM-20E, para la medición de $\mathrm{pH}$.

- Espectrofotómetro Visible Spectronic 20 Genesys Thermo scientific, para medir Absorbancia.

- Fotorreactor de diseño propio, equipado con tres lámparas Ilumedic TUV-15WG13 de radiación UV- C (Figura 1). Consiste de una caja rectangular de madera de dimensiones $50 \mathrm{~cm} \times 34 \mathrm{~cm} \times 37 \mathrm{~cm}$, forrada con cinta adhesiva de aluminio con la finalidad de reflejar la mayor cantidad de luz UV-C hacia la muestra. Dentro de la cual, se ubica en la parte superior (tapa) una lámpara, mientras que en los costados más anchos se encuentran dos lámparas, las tres Ilumedic TUV15WG13, un vaso de precipitado PYREX de $250 \mathrm{ml}$ es colocado al centro, donde la muestra (colorante y reactivos) fue agitada por medio de un agitador magnético. Un interruptor de cuchilla ubicado en la parte exterior del Fotorreactor para control de la energía a las lámparas. 


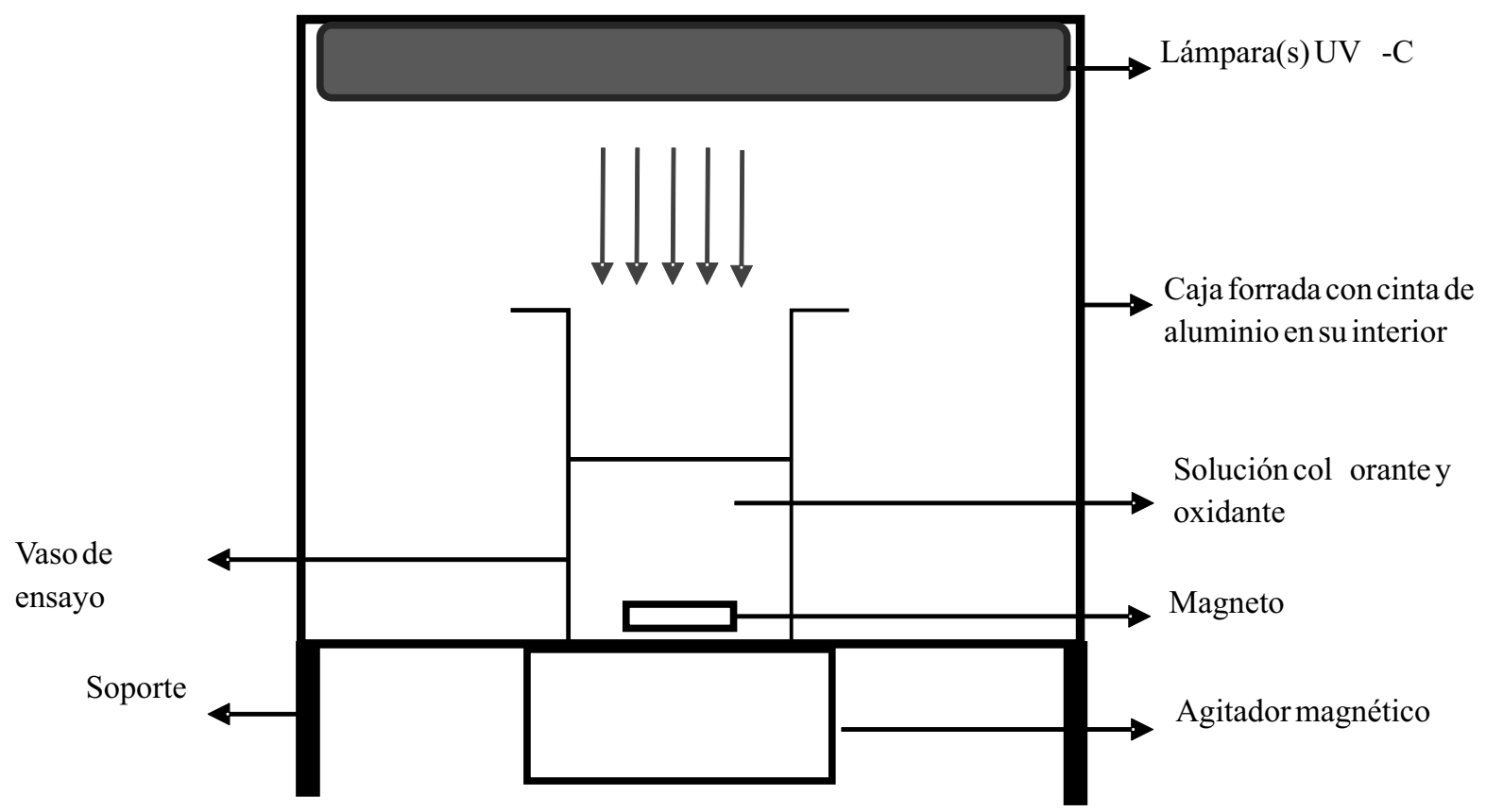

Figura 1. Fotorreactor empleado para el proceso Fotofenton y $\mathrm{H}_{2} \mathrm{O}_{2} / \mathrm{UV}$

\section{Parte Experimental}

Se preparan soluciones de colorante reactivo amarillo Sunfix S4GL (CAS) a una concentración de 20 ppm. Se acondiciona la muestra a un $\mathrm{pH}$ aproximado de 2.5 a 3, usando $\mathrm{H}_{2} \mathrm{SO}_{4}$ y NaOH diluidos.

El vaso de precipitado conteniendo la muestra y el agitador magnético son ubicados en la parte central del Fotorreactor. Se procede a agregar la solución de $\mathrm{FeSO}_{4} \cdot 7 \mathrm{H}_{2} \mathrm{O}$ y/o solución de $\mathrm{H}_{2} \mathrm{O}_{2}$, según corresponda de acuerdo al método utilizado. Se tapa el Fotorreactor y se enciende la luz UV-C, empezando a tomar el tiempo $\mathrm{t}=0$. Para el control analítico se retira una muestra de $4 \mathrm{ml}$, se lee la absorbancia de la muestra retirada, con el espectrofotómetro a la longitud de onda seleccionada y luego es guardada para su posterior regreso a la muestra original total $\left(\mathrm{m}_{\mathrm{T}}\right)$. Después de cierto tiempo, se apaga la fuente de luz y retira otra muestra $\left(\mathrm{m}_{1}\right)$ de $4 \mathrm{ml}$ e inmediatamente se regresa la muestra sacada en un inicio $\left(\mathrm{m}_{0}\right)$. Se tapa el Fotorreactor, se enciende la luz y empieza nuevamente a correr el tiempo. Finalmente, se lee la absorbancia de la muestra $\left(\mathrm{m}_{1}\right)$ y guarda para su regreso. El proceso se repite según el tiempo programado hasta que la lectura de la absorbancia se mantenga constante. Al término de la experiencia se mide el $\mathrm{pH}$ de la muestra total $\left(\mathrm{m}_{\mathrm{T}}\right)$.

\section{RESULTADOS Y DISCUSIÓN}

A los datos experimentales obtenidos se le realizó la interpretación de manera conjunta como sigue:

\section{Proceso Fotofenton}

Concentración de $\mathrm{Fe}^{+2}$ : La concentración de $\mathrm{Fe}^{+2}$ es un parámetro importante en la decoloración del CAS. Se trabaja con una concentración de $0,1,76,3,52$, y $5,28 \mathrm{mg} / \mathrm{LFe}+2$.

Se obtienen resultados (Figura 2) similares de porcentaje de decoloración, 97\% a los 15 min cuando las concentraciones de $\mathrm{Fe}^{+2}$ son 3,52 y $5,28 \mathrm{mg} / \mathrm{L}$, mientras que para 0 y $1,76 \mathrm{mg} / \mathrm{L}$ se obtuvo 73 y $79 \%$, respectivamente. Se demuestra que la cantidad de catalizador necesaria para la decoloración es un valor que se encuentra en el rango de unos pocos $\mathrm{mg} / \mathrm{L}$.

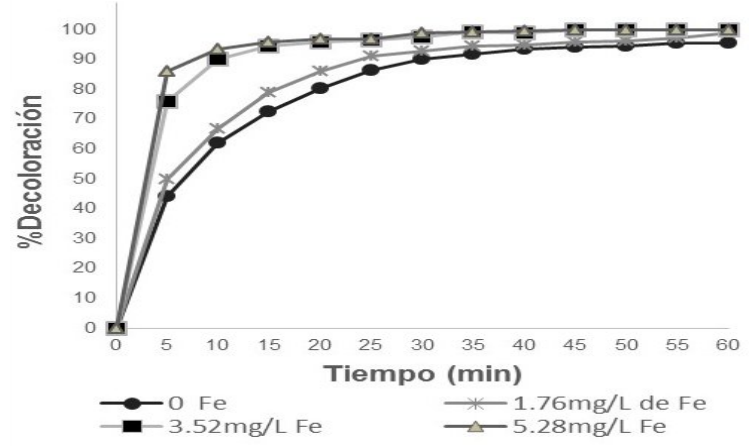

Figura 2. Efecto de la adición de $\mathrm{Fe}^{+2}$ manteniendo constante $[\mathrm{CAS}]=20 \mathrm{ppm},\left[\mathrm{H}_{2} \mathrm{O}_{2}\right]=300 \mathrm{mg} / \mathrm{L} ; \mathrm{pH}=2.51$ y 3 lámparas UV-C. 
Concentración de $\mathrm{H}_{2} \mathrm{O}_{2}$ : A partir de la concentración óptima de catalizador $\left(\mathrm{Fe}^{+2}\right)$ considerada $3,52 \mathrm{mg} / \mathrm{L}$, se evalúa la concentración de $\mathrm{H}_{2} \mathrm{O}_{2}$ necesaria para la decoloración del CAS a $20 \mathrm{ppm}$. Se trabaja a concentraciones de 100, 200, 300,400 mg/L de $\mathrm{H}_{2} \mathrm{O}_{2}$, manteniendo los demás parámetros constantes.

Los resultados obtenidos se muestran en la Figura 3 donde se observa que a los 10 min de exposición del colorante al método se obtuvo cerca al 93\% de decoloración para 200 y $300 \mathrm{mg} / \mathrm{L}$.

A los $20 \mathrm{~min}$ no existe diferencia notoria en el porcentaje de decoloración para las diferentes concentraciones de $\mathrm{H}_{2} \mathrm{O}_{2}$ llegando a una decoloración del $97 \%$ y llegando cerca al $99 \%$ a los $35 \mathrm{~min}$. Se deben tomar en cuenta los aspectos costo-beneficio respecto a las dosis de $\mathrm{H}_{2} \mathrm{O}_{2}$, ya que no se observa una gran diferencia en la decoloración entre ellas.

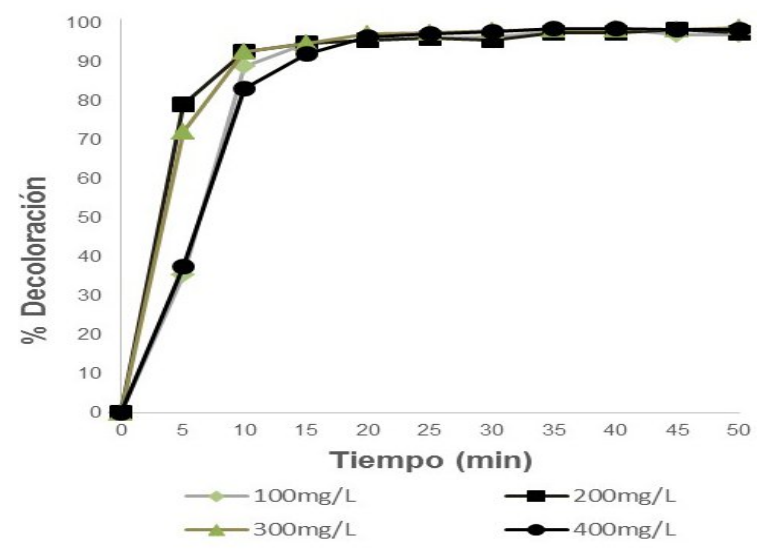

Figura 3. Efecto de la adición de $\mathrm{H}_{2} \mathrm{O}_{2}$ manteniendo constante $[\mathrm{CAS}]=20 \mathrm{ppm},\left[\mathrm{Fe}^{+2}\right]=3,52 \mathrm{mg} / \mathrm{L} ; \mathrm{pH}=2,51 ; 3$ lámparas UVC.

Efecto del pH: Observamos en la Figura 4 un pH 2,51, y que el porcentaje de decoloración llega a un 100\% en $45 \mathrm{~min}$, al aumentar el $\mathrm{pH}$ a 2,77 y 3,00, el porcentaje de decoloración disminuye necesitando más tiempo para la decoloración. Cuando el $\mathrm{pH}$ del colorante aumenta se observa una disminución del porcentaje de decoloración debido a la menor producción de radicales hidroxilos por la hidrólisis del catalizador.

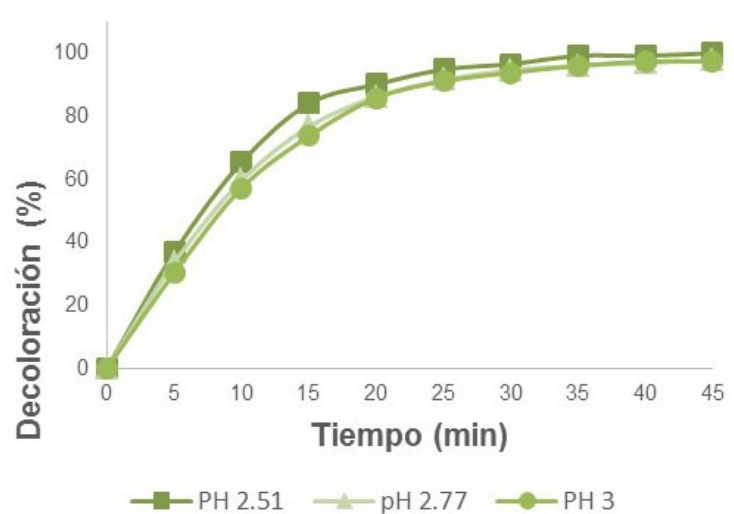

Figura 4. Efecto de la variación del $\mathrm{pH}$, manteniendo constante $[\mathrm{CAS}]=20 \mathrm{ppm},\left[\mathrm{Fe}^{+2}\right]=0,176 \mathrm{mg} / \mathrm{L} ;\left[\mathrm{H}_{2} \mathrm{O}_{2}\right]=200 \mathrm{mg} / \mathrm{L} \mathrm{y} 3$ lámparas UV-C.

Efecto de la potencia de radiación UV-C: Para determinar la influencia de la potencia de radiación necesaria para el método se evaluó a 1, 2 y 3 lámparas, y con las tres opciones se llega 100\% de decoloración. Sin embargo, se observa (Figura 5) que la diferencia respecto al tiempo en el uso de 2 o 3 lámparas es mínima. Mientras que para 1 lámpara el tiempo de decoloración es mayor. Se decide trabajar con dos lámparas por temas de costo-beneficio.

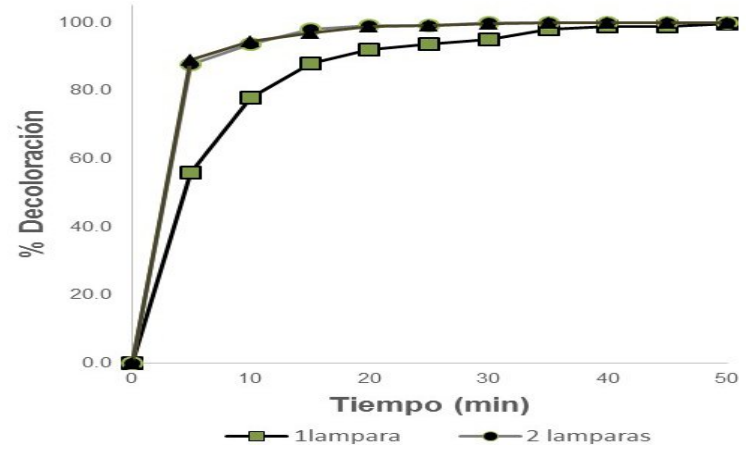

Figura 5. Efecto de la potencia de radiación UV-C, manteniendo constante $[\mathrm{CAS}]=20 \mathrm{ppm},\left[\mathrm{Fe}^{+2}\right]=3,52 \mathrm{mg} / \mathrm{L} ;\left[\mathrm{H}_{2} \mathrm{O}_{2}\right]=$ $200 \mathrm{mg} / \mathrm{L} ; \mathrm{pH}=2,51$

\section{Proceso $\mathrm{H}_{2} \mathrm{O}_{2} / \mathrm{UV}$}

Los parámetros que influyen en la decoloración del colorante CAS son el pH, la concentración del colorante CAS, $\mathrm{H}_{2} \mathrm{O}_{2}$ y la radiación $\mathrm{UV}-\mathrm{C}$.

Efecto del pH: Se investigó el efecto del pH en un rango de 3,0 a 11,0. Para ello se evaluó el porcentaje de decoloración a distintos valores de $\mathrm{pH}$. Los resultados se muestran en la Figura 6, donde observamos que el porcentaje de decoloración se incrementa proporcionalmente a medida que el $\mathrm{pH}$ disminuye. Generalmente, el pH óptimo del método $\mathrm{H}_{2} \mathrm{O}_{2} / \mathrm{UV}$ se encuentra en 
el rango 3,0 a 4,0, porque a medida que el medio es más alcalino, el peróxido de hidrógeno tiene la tendencia a descomponerse en oxígeno molecular y agua. Así, la cantidad generada de radicales $\mathrm{OH}$ disminuye haciendo el método menos eficiente. Por otra parte, la decoloración y la velocidad de reacción son limitados a $\mathrm{pH}$ bajo $(\mathrm{pH}<3,0)$ porque los radicales $\mathrm{OH}$ son consumidos por un exceso de iones $\mathrm{H}^{+}$.

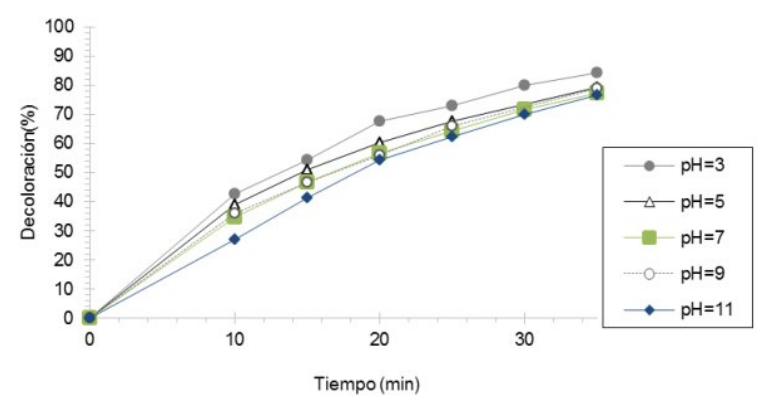

Figura 6. Efecto del $\mathrm{pH}$ manteniendo constante potencia de radiación UVC, $\left[\mathrm{H}_{2} \mathrm{O}_{2}\right]=1,47 \times 10^{-3} \mathrm{~mol} / \mathrm{L}$; $[\mathrm{CAS}]=20 \mathrm{ppm}$

Concentración de $\mathrm{H}_{2} \mathrm{O}_{2}$ : Se puede observar en la Figura 7 que la velocidad de decoloración del colorante CAS se incrementa a medida que la concentración del Peróxido de hidrógeno aumenta hasta un valor máximo, y luego disminuye. Se observa que el valor máximo de decoloración se da utilizando una concentración de $3,8 \times 10^{-2} \mathrm{~mol} / \mathrm{L} \mathrm{de} \mathrm{H}_{2} \mathrm{O}_{2}$.

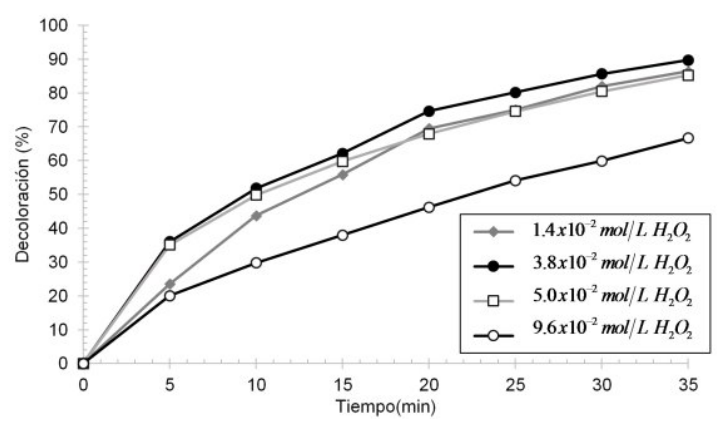

Figura 7. Efecto de la concentración del $\mathrm{H}_{2} \mathrm{O}_{2}$ manteniendo constante potencia de radiación $\mathrm{UV}-\mathrm{C},[\mathrm{CAS}]=20 \mathrm{ppm}$; $\mathrm{pH}=3$

Sin embargo, a mayor concentración de peróxido de hidrógeno se predijo que habría una mayor cantidad de radicales $\mathrm{OH}$ aumentando la eficiencia. No obstante, esta hipótesis no se ve reflejada en los resultados, ya que usando $9.6 \times 10^{-2} \mathrm{~mol} / \mathrm{L}$ de $\mathrm{H}_{2} \mathrm{O}_{2}$, que es una cantidad de $\mathrm{H}_{2} \mathrm{O}_{2}$ mayor a $3.8 \times 10^{-2} \mathrm{~mol} / \mathrm{L}$, no obtuvimos mejores resultados.
Este comportamiento se debe a que cuando existe una concentración alta de $\mathrm{H}_{2} \mathrm{O}_{2}$, y ésta va a actuar como un secuestrador de radicales $\mathrm{OH}$, mientras que a bajas concentraciones de $\mathrm{H}_{2} \mathrm{O}_{2}$, se van a generar menos radicales hidroxilo, que van a ser consumidos por el colorante y consecuentemente bajará la tasa de reacción de oxidación.

Efecto de la potencia de la radiación UV-C: Tiene efectos positivos sobre la decoloración del colorante. Este fenómeno se explica porque una alta potencia de radiación genera una mayor concentración de radicales hidroxilos, ya que la generación es directamente proporcional a la potencia de radiación UV-C.

Esta hipótesis es comprobada con los resultados mostrados en la Figura 8. En efecto, al incrementar la intensidad de radiación UV-C sobre la muestra con más lámparas se observa un mayor nivel de decoloración.

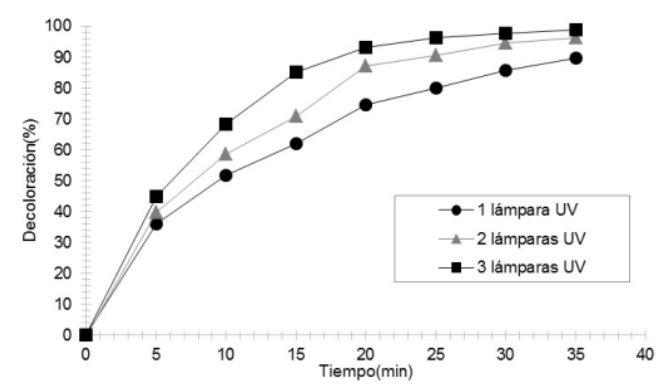

Figura 8. Efecto de la radiación UVC, expuesto a 1, 2 y 3 lámparas UV de $15 \mathrm{~W}$ cada una, manteniendo constante $\left[\mathrm{H}_{2} \mathrm{O}_{2}\right]=3,8$ $\mathrm{x} 10^{-2} \mathrm{~mol} / \mathrm{L} ;[\mathrm{CAS}]=20 \mathrm{ppm} ; \mathrm{pH}=3$.

Efecto de la concentración del colorante CAS: Entre 20 y 100 ppm (Figura 9). Se observa que el efecto es inverso, pues a mayor concentración de colorante se logra un menor porcentaje de decoloración del colorante. Esto se debe al hecho de que al haber una mayor concentración del colorante la densidad óptica interna aumenta y, consecuentemente, la permeabilidad de la solución a la radiación UV disminuye. Por ende, existirá una menor concentración de peróxido de hidrógeno que será irradiado y, con ello, la generación de radicales $\mathrm{OH}$ disminuirá. 


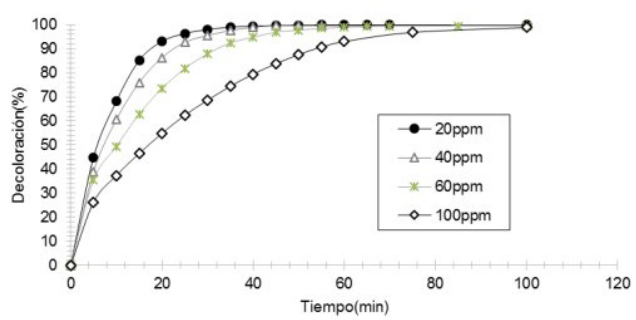

Figura 9. Efecto de la concentración del colorante CAS, manteniendo constante potencia de radiación, $\left[\mathrm{H}_{2} \mathrm{O}_{2}\right]=3.8 \times 10^{-2} \mathrm{~mol} / \mathrm{L} ; \mathrm{pH}=3$.

\section{CONCLUSIONES}

Es posible la decoloración del Colorante amarillo Sunfix S4GL (CAS). Los valores óptimos aplicando el método $\mathrm{H}_{2} \mathrm{O}_{2} / \mathrm{UV},\left[\mathrm{H}_{2} \mathrm{O}_{2}\right]=3,8 \times 10^{-2} \mathrm{~mol} / \mathrm{L}=1292$ $\mathrm{mg} / \mathrm{L} ; \mathrm{pH}=3$ y 3 lámparas $\mathrm{UV}-\mathrm{C}$, con los cuales se consiguió una decoloración del 100\% en 40 min. Los valores óptimos hallados aplicando el método Fotofenton son $\left[\mathrm{Fe}^{+2}\right]=3,52 \mathrm{mg} / \mathrm{L},\left[\mathrm{H}_{2} \mathrm{O}_{2}\right]=300 \mathrm{mg} / \mathrm{L}$, $\mathrm{pH}=2,51$ y 2 lámparas UV-C, con los cuales se consiguió una decoloración del 100\% en 30 min. El método Fotofenton es más eficiente que el $\mathrm{H}_{2} \mathrm{O}_{2} / \mathrm{UV}$ pues emplea menor cantidad de $\mathrm{H}_{2} \mathrm{O}_{2}$, menor potencia de radiación y logra la decoloración del 100\% en menor tiempo.

\section{REFERENCIAS BIBLIOGRÁFICAS}

Abdel-Alim, W., A. William. 2008. "UV-induced decolourization of acid alizarine violet $\mathrm{N}$ by homogeneous advanced oxidation processes. Chemical Engineering Journal 137 (1): 525-528.

Aleboyeh, A., Y. Moussa, y H. Aleboyeh. 2005. The effect of operational parameters on $\mathrm{UV} / \mathrm{H}_{2} \mathrm{O}_{2}$ decolourisation of Acid Blue 74. Dyes and Pigments 66 (2): 129-134.

Al-Kdasi, A., A. Idris, K. Saed y C. Teong. 2004. "Treatment of textile wastewater by advanced oxidation processes-a review." Global Nest: the International Journal 6 (3): 222-230.

Castro-Peña L., L. Durán-Herrera. 2013. “Degradación y decoloración de agua contaminada con colorantes textiles mediante procesos de oxidación avanzada." Tecnología en Marcha 27 (2): 40-5.
Fung, P. C., Q. Huang, S. M. Tsui, y C. S. Poon. 1999. "Treatability study of organic and colour removal in desizing/dyeing wastewater by UV/US system combined with hydrogen peroxide." Water Science \& Technology 40 (1): 153-160.

Juang, R. S., R. L. Tseng, F. C. Wu, y S. H. Lee. 1997. "Adsorption behavior of reactive dyes from aqueous solutions on chitosan" J. Chem. Technol. Biotechnol 70 (4): 391-399.

Kalt A., y C. Galindo. 1999. " $U V / \mathrm{H}_{2} \mathrm{O}_{2}$ oxidation of azodyes in aqueous media: evidence of a structure-degradability relationship." Dyes and Pigments 42 (3): 199-207.

Katsumata, H., S. Koike, S. Kaneco, T. Suzuki, K. Ohta. 2009. "Degradation of Reactive Yellow 86 with photo-Fenton process driven by solar light." Journal of Environmental Sciences 22 (9): 1455-1461.

Lucas M. S. y J. A. Peres. 2006. "Decolorization of the azo dye Reactive Black 5 by Fenton and photo-Fenton oxidation." Dyes and Pigments $71(3): 236-244$

Muruganandham, M. y M. Swaminathan. 2004. "Decolorization of reactive orange 4 by Fenton and photo-Fenton oxidation technology." Dyes and Pigments 63 (3): 315-321.

Novaka, N., A. Majcen Le Marecha, y M. Bogataj. 2009. "Determination of cost optimal operating conditions for decoloration and mineralization of C. I. Reactive Blue 268 by $\mathrm{UV} / \mathrm{H}_{2} \mathrm{O}_{2}$ process." Chemical Engineering Journal 151 (3): 209-219.

Pierce J. 1994. "Color in textile effluents: the origins of problem." Journal of the Society of Dyers and Colourists 110 (4): 131-133.

Rosa, J. M., A. M. F. Fileti, E. B. Tambourgi y J. C. C. Santana. 2014. "Dyeing of cotton with reactive dyestuffs: the continuous reuse of textile wastewater effluent treated by $\mathrm{UV} / \mathrm{H}_{2} \mathrm{O}_{2}$ homogeneous photocatalysis." Journal of Cleaner Production 90 (1): 60-65. 
Tuhkanen, T. A. 2004. "UV/ $\mathrm{H}_{2} \mathrm{O}_{2}$ processes." En Advanced Oxidation Processes for Water and Wastewater Treatment. Tunbridge W. y C. Pandstow (eds). London (UK): IWA Publishing. 